\title{
Rigged Hilbert Spaces in Quantum Mechanics*
}

\author{
J. E. RoBerts \\ Emmanuel College, Cambridge \\ Received April 20, 1966
}

\begin{abstract}
It is shown how rigged Hilbert spaces may be constructed in quantum mechanics, and the properties of the resulting spaces are derived. The theory is applied to non-relativistic quantum systems of $n$ interacting particles. The spectral theory in rigged Hilbert spaces is developed and the results necessary for the application to the Dirac formalism are derived.
\end{abstract}

\section{Introduction}

It has been shown in a previous paper [1] how the concept of a rigged Hilbert space provides a natural framework for introducing the Dirac bra and ket spaces into quantum mechanics. It was further shown how the introduction of extra structure into the Hilbert space by labelling certain of the observables not only provided a description of the differences between different physical systems, but also allowed a natural construction of a rigged Hilbert space associated with that quantum system. It is the purpose of this paper to go into the details of this construction and to deduce certain general topological properties of the spaces constructed in this way. To this end the actual interpretation in terms of labelled observables is irrelevant, and it is sufficient to have a suitable set of distinguished operators on Hilbert space, which are to be made continuous. This abstract theory is applied to the non-relativistic theory of $n$ interacting particles to derive necessary and sufficient conditions on the potential, so that a rigged Hilbert space may be constructed by applying the canonical method.

As the descriptions of the spectral theory in rigged Hilbert spaces are rather scattered throughout the mathematical literature and no one source gives a description which is reasonably elementary and of sufficient generality, it seemed worthwhile devoting the second part of this paper to a brief exposition of this theory. The theory is presented in the context of a spectral theory for semiinner product spaces as this makes for little additional complication and would in any case be necessary for

* The research reported in this document has been sponsored in part by the Air Force Office of Scientific Research under Grant AF EOAR 65-36, through the European Office of Aerospace Research (OAR), United States Air Force. 
applications to quantum field theory. The result of this work is to associate integral eigendecompositions with continuous observables, thus providing the necessary results for applications to the Dirac formalism.

\section{Initial topologies}

Definition 1. Let $E$ be a set and $\tau_{1}$ and $\tau_{2}$ two topologies on $E$; then we say that $\tau_{1}$ is finer than $\tau_{2}$ (or $\tau_{2}$ is coarser than $\tau_{1}$ ) and write $\tau_{1} \leqq \tau_{2}$, if each subset of $E$ which is open with respect to $\tau_{2}$ is also open with respect to $\tau_{1}$.

In a finer topology we have more open sets, more closed sets, more neighbourhoods of a given point and more continuous functions. The relation $\leqq$ introduced above is a partial ordering and allows us to give a meaning to the terms lower bound and upper bound, when referring to topologies. It is a feature of the set of topologies on a given set that it forms a complete lattice with respect to this partial ordering. In other words, every subset of topologies on a given set has a greatest lower bound and a least upper bound. This allows the following construction.

Definition 2. Let $T_{\alpha}: E \rightarrow E_{\alpha}(\alpha \in A)$ be mappings of $E$ into topological spaces $E_{\alpha}$; then the coarsest topology on $E$ making the mappings $T_{\alpha}(\alpha \in A)$ continuous is called the initial topology with respect to the mappings $T_{\alpha}$.

The nomenclature followed here is that of BoURBAKI [2]; other terms are Kerntopologie [3] and projective topology [4]. The initial topology may be characterized explicitly as follows: if $B_{\alpha}(\alpha \in A)$ are bases for the topologies on $E_{\alpha}$, then the set of finite intersections of sets of the form $T_{\alpha}^{-1}\left(U_{\alpha}\right)\left(U_{\alpha} \in B_{\alpha}, \alpha \in A\right)$ forms a basis for the initial topology on $E$. This topology has the following fundamental property.

Proposition 1. Let $E$ have the initial topology with respect to the mappings $T_{\alpha}: E \rightarrow E_{\alpha}(\alpha \in A)$ and let $S: F \rightarrow E$ be a mapping from a topological space $F$ into $E$, then $S$ is continuous if and only if $T_{\alpha} S: F \rightarrow E_{\alpha}$ is continuous for all $\alpha \in A$.

This is a standard result in general topology [2] from which we immediately deduce

Proposition 2. (Transitivity of the initial topology.) Let $T_{\alpha}: E \rightarrow E_{\alpha}$ $(\alpha \in A)$ be mappings between sets $E$ and $E_{\alpha}$, and $T_{\beta_{\alpha}}: E_{\alpha} \rightarrow E_{\beta_{\alpha}}\left(\beta_{\alpha} \in B_{\alpha}\right)$ be mappings into topological spaces $E_{\beta_{\alpha}}$. Then the initial topology on $E$ with respect to the mappings $T_{\beta_{\alpha}} T_{\alpha}: E \rightarrow E_{\beta_{\alpha}}$ is the same as the initial topology with respect to $T_{\alpha}: E \rightarrow E_{\alpha}$, if each $E_{\alpha}$ is given its initial topology with respect to the mappings $T_{\beta_{\alpha}}: E_{\alpha} \rightarrow E_{\beta_{\alpha}}$.

Analogous results hold when the sets $E, E_{\alpha}$ have additional structure and the maps $T_{\alpha}$ are structure preserving. We shall need these results when $E$ and $E_{\alpha}$ are some sort of topological vector space, and the key to obtaining them is contained in the following proposition. 
Proposition 3. Let $T_{\alpha}: E \rightarrow E_{\alpha}(\alpha \in A)$ be linear maps of a linear space $E$ into topological vector spaces $E_{\alpha}$; then

a) The initial topology on $E$ makes $E$ a topological vector space.

b) If $E_{\alpha}$ is locally convex $(\alpha \in A)$ so is $E$ [5].

c) If $E_{\alpha}$ is a Schwartz space $(\alpha \in A)$ so is $E$ [4].

d) If $E_{\alpha}$ is a nuclear space ( $\alpha \in A$ ) so is $E$ [6], [7].

It must be remarked here, that in no case do we take the definitions of these classes of topological vector spaces to imply that they are Hausdorff. We need only notice that the trivial topology on $E$, in which the only open sets are the empty set and the whole space, makes $E$ a nuclear space, and we have deduced

Proposition 4. The set of nuclear, Schwartz, locally convex or topological vector space topologies on a linear space $E$ each forms a complete sublattice of the complete lattice of all topologies on $E$ in which the greatest lower bound coincides with the topological one.

Proposition 5. Let $E_{\alpha}$ be Hausdorff topological vector spaces; then the initial topology on $E$ with respect to linear mappings $T_{\alpha}: E \rightarrow E_{\alpha}$ is Hausdorff if and only if, for each $x \in E$, there exists an $\alpha \in A$ such that $E_{\alpha} x \neq 0$.

If the initial topology does satisfy the conditions of the above proposition, we shall write $E=K T_{\alpha}^{-1}\left(E_{\alpha}\right)$.

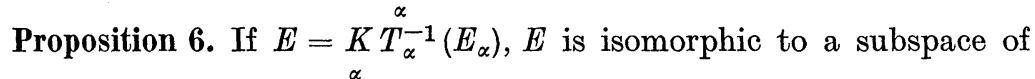
$\Pi E_{\alpha}$.

Proof. Define a mapping $T: E \rightarrow \prod E_{\alpha}$ by setting $T x=\left(T_{\alpha} x\right), T$ is $1-1$. By proposition $2, E$ has the coarsest topology making $T$ continuous, and $E$ is thus isomorphic to the subspace $T(E)$ of $\Pi E_{\alpha}$.

So far, we have just given a sketch of the standard theory of initial topologies, but for applications to quantum mechanics the theory needs certain modifications and refinements. In the first place, $E$ already has a topology, which must be taken into account, so that we are led to give $E$ the coarsest topology finer than the original topology and making the mappings $T_{\alpha}: E \rightarrow E_{\alpha}$ continuous. This situation may be reduced to the previous case simply by including the identity mapping $I: E \rightarrow E$ in the set $\left\{T_{\alpha}\right\}_{\alpha} \in A_{A}$. Secondly, the operators we shall eventually be dealing with will be defined on dense domains and will be derived from closed operators.

Definition 3. Let $T$ be a mapping between topological spaces $E$ and $F$ with domain of definition $D(T)$, then $T$ is said to be a closed mapping if the set $\{(x, T x) \mid x \in D(T)\}$, the graph of $T$, is a closed subset of $E \times F$. 
This concept must be generalized.

Definition 4 . We call a set of mappings $\left\{T_{\alpha}\right\}_{\alpha \in A}$ between $E$ and $E_{\alpha}$, defined on a common subspace $D \subset E$ and containing the identity $I: E \rightarrow E$ restricted to $D$, a closed set if $\left\{\left(T_{\alpha} x\right) \mid x \in D\right\}$ is closed in $\Pi E_{\alpha}$. If $\left\{T_{\alpha}\right\}_{\alpha} \in A_{A}$ does not contain $I \mid D$, we call it closed if the set obtained by adjoining this mapping is closed.

This definition includes the previous one as a special case.

Proposition 7. Suppose $T_{\alpha}$ has domain $D_{\alpha} \subset E$ and is closed $(\alpha \in A)$, and let $D=\bigcap_{\alpha} D_{\alpha}, \dot{T}_{\alpha}=T_{\alpha} \mid D$; then $\left\{\dot{T}_{\alpha}\right\}_{\alpha \in A}$ is a closed set of mappings.

Proof. As $T$ is closed, $\left\{\left(x, T_{\alpha} x\right) \mid x \in D\right\}$ is closed in $E \times E_{\alpha}$. Thus $\left\{\left(x, T_{\alpha} x\right) \mid x \in D_{\alpha}\right\} \times \prod_{\beta \neq \alpha} E_{\beta}$ is closed in $E \times \prod_{\beta \in A} E_{\beta}$. It follows that $\left\{\left(x,\left(T_{\alpha} x\right)\right) \mid x \in D, \alpha \in A\right\}$ is closed in $E \times \prod_{\alpha \in A} E_{\alpha}$, as it is the intersection of the above closed sets. Hence $\left\{\tilde{T}_{\alpha}\right\}_{\alpha} \in A$ is indeed closed.

Working with closed sets of operators leads to many regularity properties of the resulting topologies, the most important of which are listed in the following proposition.

Proposition 8. Let $\left\{T_{\alpha}\right\}_{\alpha \in A}$ be a closed set of operators with identity, and let $F=K T_{\alpha}^{-1}\left(E_{\alpha}\right)$; then

a) If $E_{\alpha}$ is complete $(\alpha \in A), F$ is also complete.

b) If $E_{\alpha}$ is semireflexive $(\alpha \in A), F$ is also semireflexive.

If $A$ is countable, we have further

c) If $E_{\alpha}$ is metrizable $(\alpha \in A), F$ is also metrizable.

d) If $E_{\alpha}$ is reflexive $(\alpha \in A), F$ is also reflexive.

e) If $E_{\alpha}$ is Fréchet ( $\left.\alpha \in A\right), F$ is also Fréchet.

Proof. These results all follow immediately from the fact that $F$ is isomorphic to a closed subspace of $\Pi E_{\alpha}[3]$.

Finally, we are really interested in the case in which the image space is the same as the domain space, and we want to have continuity even when we change the topologies in both spaces at once. This is achieved in the following proposition.

Proposition 9. Let $\left\{T_{\alpha}\right\}_{\alpha \in A}$ be a semigroup of operators on a topological space $E$. If $F$ denotes the space $E$ with the initial topology with respect to the mappings $T_{\alpha}: E \rightarrow E(\alpha \in A)$, then each $T_{\alpha}$ induces a continuous mapping $T_{\alpha}: F \rightarrow F$.

Proof. By Proposition 1, a mapping $T_{\beta}: F \rightarrow F$ is continuous if and only if $T_{\alpha} T_{\beta}: F \rightarrow E$ is continuous, for all $\alpha \in A$. However, $T_{\alpha} T_{\beta} \in$ $\left\{T_{\gamma}\right\}_{\gamma \in A}$, so the condition is fulfilled. 
For the sake of completeness we remark that many of the function spaces used in functional analysis may be regarded as having been constructed by making a closed set of mappings continuous [8]. The duals of these spaces include many distribution spaces, which may thus be regarded as having been constructed in this manner. Alternatively we may construct these spaces of distributions using the dual method of final topologies [2], which involves changing the topology on the image rather than the domain space.

\section{Applications to Hilbert space}

We suppose given a Hilbert space $\mathscr{H}$ and a set $\mathcal{O}$ of closed operators on $\mathscr{H}$ with the property that if $A \in \mathcal{O}, A^{*} \in \mathcal{O}$.

Proposition 10. If $\mathcal{O}$ has a dense invariant domain, then $\mathcal{O}$ generates a *-algebra of closed operators with identity, $\mathscr{A} \supset \mathcal{O}$, where tors.

(i) Scalar multiplication is the usual scalar multiplication of opera-

(ii) Addition is the strong sum [9], the closure of the usual sum of operators.

(iii) Multiplication is the strong product, the closure of the usual product of operators.

The maximal invariant domain for $\mathcal{O}$ is given by $D=\bigcap_{A \in \mathscr{A}} D(A)$, and $\mathscr{A}^{0} \equiv \mathscr{A} \mid D$ is just the algebra with unit generated by $\mathscr{O}^{0} \equiv \mathcal{O} \mid D$.

Proof. It suffices to note that the existence of a dense invariant domain always guarantees the existence of adjoints. The fact that $\mathcal{O}$ is *-closed shows that these adjoints leave the invariant domain invariant. The closure of an operator $A$ is $A^{* *}$.

We want to make $\mathcal{O}^{0}$ into a set of continuous operators on $D$ by giving $D$ a suitable topology. For the purposes of discussion we shall say that a topology $\tau$ on $D$ is allowed if $\mathcal{O}^{0}$ is continuous $\mathcal{O}^{0}: D_{\tau} \rightarrow D_{\tau}$ and if $\tau \leqq \tau_{\mathscr{H}}$, where $\tau_{\mathscr{H}}$ is the topology on $D$ induced by the Hilbert space $\mathscr{H}$. Let $\tau_{i}$ denote the initial topology with respect to the mappings $\mathscr{A}^{0}: D \rightarrow \mathscr{H}$.

Proposition 11. $\tau_{i}$ is the coarsest allowed topology.

Proof. $\mathscr{A}^{0}: D_{\tau_{i}} \rightarrow \mathscr{H}$ is continuous by definition. $\mathscr{A}^{0}$ is an algebra and hence, a priori, a semigroup, so that by Proposition $9 \mathscr{A}^{0}: D_{\tau_{i}} \rightarrow D_{\tau_{i}}$ is continuous. Further $I \mid D \in \mathscr{A}^{0}$ so that $\tau_{i} \leqq \tau_{\mathscr{H}}$ and $\tau_{i}$ is allowed. If $\tau$ is allowed then $\mathcal{O}^{0}: D_{\tau} \rightarrow D_{\tau}$ is continuous and thus $\mathscr{A}^{0}: D_{\tau} \rightarrow D_{\tau}$ is continuous. Hence a priori $\mathscr{A}^{0}: D_{\tau} \rightarrow \mathscr{H}$ is continuous, for $\tau \leqq \tau_{\mathscr{H}}$. It follows that $\tau \leqq \tau_{i}$ so that $\tau_{i}$ is indeed the coarsest allowed topology.

We shall take $\tau_{i}$ as our topology for $D$ and denote the resulting topological vector space by $\Phi$. The completion of $\Phi$ with respect to the inner product topology is $\mathscr{H}$, as we have assumed $D$ to be dense in $\mathscr{H}$. Let $T$ denote the canonical embedding $T: \Phi \rightarrow \mathscr{H}$. Its adjoint $T^{\times}$is a 
continuous linear mapping $T^{\times}: \mathscr{H}^{\times} \rightarrow \Phi_{b}^{\times}$defined by $\langle T \phi, x\rangle$ $=\left\langle\phi, T^{\times} x\right\rangle(\phi \in \Phi, x \in \mathscr{H})$. Here $\Phi_{b}^{\times}$denotes the space of continuous antilinear functionals on $\Phi$ with the strong topology of the dual system $\left\langle\Phi, \Phi^{\times}\right\rangle[4]$.

Proposition 12. $T^{\times}$is $1-1$ and $T^{\times}\left(\mathscr{H}^{\times}\right)$is dense in $\Phi^{\times}$with respect to any topology on $\Phi^{\times}$compatible with the dual system $\left\langle\Phi, \Phi^{\times}\right\rangle$.

Proof. $T$ is $1-1$ and maps onto a dense subspace of $\mathscr{H}$. The result follows from the well-known properties of adjoint mappings [4].

There is a natural isomorphism $\mathscr{H} \cong \mathscr{H}^{\times}$and after identifying these spaces we get a canonical triplet of spaces $\Phi \subset \mathscr{H} \subset \Phi_{b}^{\times}$associated with each space $\Phi$ generated in this way.

Proposition 13. Let $\Phi$ be a space constructed as above, then

a) The topology on $\Phi$ is given by the seminorms $\phi \rightarrow\left\|A^{0} \phi\right\|$ where $A^{0}$ runs through $\mathscr{A}^{0}$.

b) $\Phi$ is complete and semireflexive.

c) If $\mathcal{O}$ is a countable set of operators, then $\Phi$ is a reflexive Fréchet space.

Proof. As $A^{0}$ is continuous and $\phi \rightarrow\|\phi\|$ is a continuous seminorm on $\Phi, \phi \rightarrow\left\|A^{0} \phi\right\|$ must also be a continuous seminorm on $\Phi$. On the other hand, if $\Phi$ has the topology given by these seminorms, $A^{0}: \Phi \rightarrow \Phi$ is indeed continuous. This proves a). Now $\mathscr{A}^{0}=\mathscr{A} \mid D$, so $\mathscr{A}^{0}$ is closed by Proposition 7, and b) and c) follow if we apply Proposition 8 noting that $\mathscr{H}$ is a reflexive Fréchet space.

We note in passing that as we have shown that $\Phi$ is semireflexive, it follows from Proposition 12 that $T^{\times} T(\Phi)$ is dense in $\Phi_{b}^{\times}$.

GEL'FAND and SILOV [10] introduce the concept of compatibility of norms, and work mainly with spaces whose topologies can be given by a sequence of pairwise compatible norms. If we construct topologies by making a set of operators with identity on Hilbert space continuous, then the resulting space has a topology given by a set of pairwise compatible norms if and only if the operators have closed extensions [8]. If we take in particular a countable set of closed operators, then we just get a countably Hilbert space in the sense of GEL'FAND and VILENKIN [11].

\section{Rigged Hilbert spaces}

We shall make frequent use of the following notation due to GrothenDIECK [4].

Definition 5. Let $A$ be an absolutely convex set in a linear space $E$, then $\Lambda(A)$ will denote the vector subspace generated by $A$. Putting $N=\{x \mid \lambda x \in A(\lambda \in \mathbf{C})\}$, we shall denote by $E_{A}$ the quotient space $\Lambda(A) / N$ equipped with the norm $\|x\|_{A}=\inf _{x \in \lambda A}|\lambda|$, and by $\widehat{E_{A}}$ the Banach space formed by completing $E_{A}$. 
Definition 6. Let $T: E \rightarrow F$ be a linear mapping of two locally convex spaces, then $T$ is compact if it maps arbitrary neighbourhoods of the origin into relatively compact sets. $T$ is nuclear [6], [7] if it may be expressed in the following form.

$$
T x=\sum_{k=1}^{\infty}\left\langle x, x_{k}^{\prime}\right\rangle y_{k}(x \in E)
$$

where $x_{k}^{\prime} \in E_{A}^{\prime}, y_{k} \in F_{B}$ and where $A$ is an equicontinuous, absolutely convex, weakly closed subset of $E^{\prime}, B$ a bounded absolutely convex subset of $F$ such that $F_{B}$ is complete, and $\sum_{k=1}^{\infty}\left\|x_{k}^{\prime}\right\|_{A}\left\|y_{k}\right\|_{B}<\infty$.

A nuclear mapping is necessarily compact and a compact mapping is necessarily continuous. For the case of mappings between Hilbert spaces, the compact mappings are the completely continuous mappings and the nuclear mappings are the mappings of trace class.

Proposition 14. A mapping $T: \mathscr{H}_{1} \rightarrow \mathscr{H}_{2}$ between two (separable) Hilbert spaces is compact if and only if it can be expressed in the form

$$
T x=\sum_{k=1}^{\infty} \lambda_{k}\left(x_{k}, x\right) y_{k}\left(x \in \mathscr{H}_{1}\right)
$$

where $\left(x_{k}\right)$ and $\left(y_{k}\right)$ are complete orthonormal sequences in $\mathscr{H}_{1}$ and $\mathscr{H}_{2}$ respectively, $\lambda_{k} \geqq 0$ and $\lambda_{k} \rightarrow 0$ as $k \rightarrow \infty$. Further $T$ is nuclear if and only if $\sum_{k=1}^{\infty} \lambda_{k}<\infty[11]$.

Definition \%. A locally convex space is a nuclear space (Schwartz space) if, given any absolutely convex neighbourhood of the origin $V$, there exists another such $U \subset V$, such that the canonical mapping $\widehat{E_{U}} \rightarrow \widehat{E_{V}}$ is nuclear (compact).

These definitions differ from those given by Grothendieck [6], [4] only in that we allow spaces which are not Hausdorff. However a locally convex space is nuclear (Schwartz) if and only if its associated Hausdorff space [5] is nuclear (Schwartz).

We now wish to decide when the space $\Phi$ constructed in the last section from the pair $(\mathscr{H}, \mathcal{O})$ is nuclear or Schwartz. We consider first the case where $\mathcal{O}$ is the set consisting of the single self-adjoint operator $A$. Now, by the spectral theorem [11], there is a spectral resolution over the real line $\mathbf{R}^{1}$ associated with $A$

$$
\mathscr{H}=\int \mathscr{H}(\lambda) d \mu(\lambda) \text {. }
$$

The domain of $A, D_{1}$ say, consists of those measurable vector fields $\phi(\cdot)$, for which

and

$$
(\phi, \phi)=\int(\phi(\lambda), \phi(\lambda))_{\lambda} d \mu(\lambda)<\infty,
$$

$$
(A \phi, A \phi)=\int \lambda^{2}(\phi(\lambda), \phi(\lambda))_{\lambda} d \mu(\lambda)<\infty .
$$


We now define $D_{n}$ to consist of those vector fields $\phi(\cdot)$, for which

$$
\left(A^{m} \phi, A^{m} \phi\right)<\infty \quad(m=0,1,2, \ldots, n) .
$$

$D$, the maximal invariant domain for the algebra generated by $A$, is just $\bigcap_{n} D_{n}$ and is thus always dense in $\mathscr{H}$, for $D$ contains all those vector fields $\phi(\cdot) \in \mathscr{H}$, which have compact support, and these fields are themselves dense in $\mathscr{H}$. Each $D_{n}$ has a natural topology as a Hilbert space with the scalar product

$$
(\phi, \psi)_{n}=\int\left[1+\lambda^{2}+\cdots+\lambda^{2 n}\right](\phi(\lambda), \psi(\lambda))_{\lambda} d \mu(\lambda)
$$

and $\Phi$ is then the topological projective limit [3] of the $D_{n}$ and its topology is given by the set of norms

$$
\phi \rightarrow \sqrt{(\phi, \phi)_{n}} \quad(n=0,1, \ldots) .
$$

Proposition 15. Let $\Phi \subset \mathscr{H} \subset \Phi^{\times}$be the canonical triplet constructed from $(\mathscr{H},\{A\})$, then $\Phi$ is a nuclear (Schwartz) space if and only if for some $n\left(I+A^{2}+\cdots+A^{2 n}\right)^{-1 / 2}$ is a nuclear (compact) mapping $\mathscr{H} \rightarrow \mathscr{H}$.

Proof. Suppose $\Phi$ is nuclear, then for some $n$ the canonical mapping $T_{n}: D_{n} \rightarrow \mathscr{H}$ must be nuclear. Let $T_{n}^{\times}$denote the adjoint of $T_{n}$. $T_{n}^{\times}: \mathscr{H} \rightarrow D_{n}$ and is defined by

\section{Hence}

$$
(\phi, \psi)=\left(T_{n}^{\times} \phi, \psi\right)_{n}\left(\phi \in \mathscr{H}, \psi \in D_{n}\right) .
$$

Thus also

$$
\left[T_{n}^{\times} \phi\right](\lambda)=\left[1+\lambda^{2}+\cdots+\lambda^{2 n}\right]^{-1} \phi(\lambda) \text {. }
$$

$$
\left[T_{n}^{\times} T_{n} \phi\right](\lambda)=\left[1+\lambda^{2}+\cdots+\lambda^{2 n}\right]^{-1} \phi(\lambda)
$$

and so $\left(T_{n}^{\times} T_{n}\right)^{1 / 2}=\left(I+A^{2}+\cdots+A^{2 n}\right)^{-1 / 2}$. But $T_{n}$ is nuclear if and only if $\left(T_{n}^{\times} T_{n}\right)^{1 / 2}$ is nuclear (see Proposition 14). Conversely suppose that, for some $n,\left[I+A^{2}+\cdots+A^{2 n}\right]^{-1 / 2}$ is nuclear, then the canonical mapping $D_{n} \rightarrow \mathscr{H}$ is nuclear, as is the canonical mapping $D_{n+m} \rightarrow D_{n}$ $(m=1,2 \ldots)$. But the sets of the form $N(n, \varepsilon)=\left\{\phi \mid\|\phi\|_{n}<\varepsilon\right\}, \varepsilon>0$, $n=1,2, \ldots$ form a base of neighbourhoods of the origin, so that $\Phi$ is nuclear.

The proof for the case of Schwartz spaces may be obtained by substituting 'compact mapping' for 'nuclear mapping' everywhere. The result implies in particular that, if $\Phi$ is to be Schwartz, then $A$ must have a purely discrete spectrum of finite multiplicity. If the eigenvalues of $A$ are $\lambda_{i}, i=1,2 \ldots$, allowing for multiplicity, then $\lambda_{i} \rightarrow \infty$, as $i \rightarrow \infty$. The condition for $\Phi$ to be nuclear is that for some $n$,

$$
\sum_{i=1}^{\infty}\left(1+\left|\lambda_{i}\right|\right)^{-n}<\infty
$$


For the general case, we take $\mathcal{O}$ to be a ${ }^{*}$-closed set of closed operators, generating a *algebra with unit $\mathscr{A}$, such that $D=\bigcap_{A \in \mathscr{A}} D(A)$ is dense in $\mathscr{H}$.

Theorem 1. Let $\Phi \subset \mathscr{H} \subset \Phi^{\times}$be the canonical triplet constructed from $(\mathscr{H}, \mathcal{O})$, then $\Phi$ is a nuclear (Schwartz) space if and only if there exists an $A^{0} \in \mathscr{A}^{0}$ having a self-adjoint extension in $\mathscr{H}$, whose inverse is a nuclear (compact) mapping.

Proof. Necessity: if $\Phi$ is nuclear (Schwartz), then for some absolutely convex neighbourhood of the origin $V$, the canonical mapping $\widehat{D_{V}} \rightarrow \mathscr{H}$ must be nuclear (compact). Now by Proposition 13, $\Phi$ has the topology given by the seminorms $\varphi \rightarrow\left\|A^{0} \phi\right\|$ as $A$ runs through $\mathscr{A}$. Hence we may choose $V$ to be of the form

$$
\left\{\phi \in D \mid \sup _{i=1}^{n}\left\|A_{i} \phi\right\|<1(i=1,2, \ldots, n)\right\}
$$

where $A_{i} \in \mathscr{A}(i=1,2, \ldots, n)$, for such sets form a base of neighbourhoods of the origin in $\Phi$. Set $B=\sum_{i=1}^{n} A_{i}^{*} A_{i}+I$, then $\bar{B} \in \mathscr{A},(\bar{B}$ the closure of $B$ ) and $\bar{B}^{0}=\sum_{i=1}^{n} A_{i}^{* 0} A_{i}^{0}+I^{0}$.

$(B \phi, B \phi)=(\phi, \phi)+\left(\sum_{i=1}^{n} A_{i}^{*} A_{i} \phi, \sum_{i=1}^{n} A_{i}^{*} A_{i} \phi\right)+2 \sum_{i=1}^{n}\left(A_{i} \phi, A_{i} \phi\right)(\phi \in D)$. Then, taking $U=\{\phi \in D \mid(B \phi, B \phi)<1\}, U \subset V$, so that $\widehat{D_{U}} \rightarrow \mathscr{H}$ is nuclear (compact). Now $\left(B^{0} \phi, B^{0} \phi\right) \geqq(\phi, \phi)(\phi \in D)$, so $\widehat{D_{U}}$ is algebraically isomorphic to $D\left(\overline{B^{0}}\right)$. Let $T$ be the canonical map $T: \widehat{D_{U}} \rightarrow \mathscr{H}$ then its adjoint $T^{\times}: \mathscr{H} \rightarrow \widehat{D_{U}}$ satisfies

Hence

$$
(\phi, \psi)=\left(T^{\times} \phi, \psi\right) \widehat{D_{U}}\left(\psi \in D\left(\overline{B^{0}}\right), \phi \in \mathscr{H}\right) .
$$

Thus

$$
(\phi, \psi)=\left(\overline{B^{0}} T T^{\times} \phi, \overline{B^{0}} \psi\right) \quad\left(\psi \in D\left(\overline{B^{0}}\right), \phi \in \mathscr{H}\right) .
$$

$$
\overline{B^{0}} T T^{\times} \phi \in D\left(\overline{B^{0}} *\right) \text { and } \overline{B^{0}} * \overline{B^{0}} T T^{\times} \phi=\phi \quad(\phi \in \mathscr{H}),
$$

and so

$$
T T^{\times}=\left(\overline{B^{0}} * \overline{B^{0}}\right)^{-1} .
$$

Now $T$ is nuclear (compact) if and only if $\left(T T^{\times}\right)^{1 / 2}$ is nuclear (compact), so that $\left(\overline{B^{0}} * \overline{B^{0}}\right)^{-1 / 2}$ is nuclear (compact). Now setting $A=\overline{B^{0}} * \overline{B^{0}}, A$ is self-adjoint and $A^{-1 / 2}$ is nuclear (compact), hence $A^{-1}$ is also nuclear (compact). Further $A^{0}=B^{* 0} B^{0} \in \mathscr{A}^{0}$ as required. 
Sufficiency: the topology on $D$ giving $\Phi$ is the coarsest making the mappings $A^{0}: D \rightarrow \mathscr{H}$ continuous $\left(A^{0} \in \mathscr{A}^{0}\right)$, and it thus depends only on the $A^{0}$ and not on their extensions. Suppose $B^{0} \in \mathscr{A}^{0}$ has a selfadjoint extension $B$ such that $B^{-1}$ is nuclear (compact). Let $E=\bigcap_{n=1}^{\infty} D\left(B^{n}\right)$, then $E \supset D$. Also $\left(B^{n}\right)^{0}=\left(B^{0}\right)^{n}$. If we give $D$ the initial topology with respect to the mappings $A^{0}: D \rightarrow E, A^{0} \in \mathscr{A}^{0}$, and $E$ the initial topology with respect to the mappings $B^{n}: E \rightarrow \mathscr{H}, n=0,1,2, \ldots$, then by Proposition $2, D$ has just its topology as the space $\Phi$. However $\left(I+B^{2}\right)^{-1 / 2}$ is nuclear (compact) so $E$ is nuclear (Schwartz) by Proposition 15. Hence, by Proposition $3, \Phi$ is also nuclear (Schwartz).

In the non-relativistic theory of $n$ interacting particles $\Phi$ will be nuclear as will be shown in the next section. This will enable us to develop a satisfactory spectral theory.

Definition 8. If $\Phi$ is nuclear, then the triplet $\Phi \subset \mathscr{H} \subset \Phi^{\times}$will be called a rigged Hilbert space.

\section{Non-relativistic quantum mechanics}

For a non-relativistic quantum system of $n$ interacting particles, where each particle has a finite number of independent internal states, there is no difficulty in showing that $\Phi$ is nuclear if we choose $\mathcal{O}$ to be a set of observables having a common invariant dense domain, provided we include the position and momentum observables of all the particles [1]. However if we wish to interpret $\mathcal{O}$ as the labelled observables of the system, we would naturally wish to include the Hamiltonian. As will now be shown, this can only be done if the potential energy is almost everywhere infinitely differentiable. The question at issue is the existence of a dense invariant domain $D$. If we suppress the internal degrees of freedom and suppose for convenience that the particles are distinguishable, then we may represent the Hilbert space $\mathscr{H}$ as $L^{2}\left(\mathbf{R}^{3 n}\right)$, the space of square integrable functions on $\mathbf{R}^{3 n}$ with respect to Lebesgue measure. If $\mathcal{O}$ includes the position and momentum variables of all the particles, then $D \subset \mathscr{S}\left(\mathbf{R}^{3 n}\right)$ [1], where $\mathscr{S}\left(\mathbf{R}^{3 n}\right)$ is the usual Schwartz space of $C^{\infty}$-functions vanishing at infinity faster than any inverse power of the distance [12]. Suppose that for those vectors, which are both in the domain of the Hamiltonian and in $\mathscr{S}\left(\mathbf{R}^{3 n}\right) \subset L^{2}\left(\mathbf{R}^{3 n}\right)$, the Hamiltonian has the form

$$
H=\sum_{i=1}^{3 n}\left(P_{i}^{2} / 2 m_{i}\right)+V\left(x_{1}, x_{2}, \ldots, x_{3 n}\right) .
$$

We need a few simple lemmas.

Lemma 1. Let $\Omega$ be an open set in $\mathbf{R}^{3 n}$, and let $\mathscr{D}(\Omega)$ denote the set of all $C^{\infty}$-functions with compact support in $\Omega$. Then $\mathscr{D}(\Omega)$ is dense in

8 Commun. math. Phys., Vol. 3 
$L^{2}\left(\mathbf{R}^{3 n}\right)$ if and only if $S=\mathbf{R}^{3 n} \sim \Omega$, the complement of $\Omega$, has zero Lebesgue measure.

Proof. $\mathscr{D}\left(\mathbf{R}^{3 n}\right)$ is certainly dense in $L^{2}\left(\mathbf{R}^{3 n}\right)$. Suppose $S$ is closed and has Lebesgue measure zero. Then given $\varepsilon$, there exists an open set $G \supset S$ with $\mu(G)<\varepsilon . G$ and $\Omega$ form an open covering of $\mathbf{R}^{3 n}$. Let $\alpha_{1}(x), \alpha_{2}(x)$ be a partition of unity subordinate to the given covering [12], with $\operatorname{supp} \alpha_{1}(x) \subset G$, and $\operatorname{supp} \alpha_{2}(x) \subset \Omega$. Given $f \in \mathscr{D}\left(\mathbf{R}^{3 n}\right), \alpha_{2} f \in \mathscr{D}(\Omega)$ and $\left\|\alpha_{1} f\right\|^{2} \leqq M^{2} \varepsilon$, where $M$ is an upper bound for $f$ on $\mathbf{R}^{3 n}$. Hence $\mathscr{D}(\Omega)$ is dense in $L^{2}\left(\mathbf{R}^{3 n}\right)$ in the sense of the $L^{2}$-norm. Conversely if $S$ has nonzero Lebesgue measure, we can find a $C^{\infty}$-function with support in $S$, which gives a non-zero element of $\mathscr{H}$. Such a function is orthogonal to $\mathscr{D}(\Omega)$, which cannot thus be dense in $L^{2}\left(\mathbf{R}^{3 n}\right)$.

Lemma 2. Let $x \in \mathbf{R}^{\mathbf{1}}$, if $f(x)$ and $f(x) g(x)$ are both differentiable at $x=x_{0}$, but $g(x)$ is not differentiable at $x=x_{0}$, then $f\left(x_{0}\right)=0$.

This is an elementary exercise in analysis, which leads to

Lemma 3. Take $x \in \mathbf{R}^{3 n}$, and let $d(x)$ be the degree of differentiability of $V(x)$ at the point $x$. Put $S=\{x \mid d(x)<\infty\}$, then if $f(x) \in \mathscr{S}\left(\mathbf{R}^{3 n}\right)$, and $V(x) f(x) \in \mathscr{S}\left(\mathbf{R}^{3 n}\right), f(x)=0(x \in \bar{S})$.

Proof. Repeated application of Lemma 2 to the various partial derivatives of $f(x)$ and $V(x) f(x)$ gives $f(x)=0$ for $x \in \bar{S}$.

Theorem 2 [1]. A necessary and sufficient condition for the labelled Hilbert space of the quantum system of $n$ interacting particles to generate a rigged Hilbert space is that the potential energy should be $C^{\infty}$ on some open set $\Omega$ whose complement has zero Lebesgue measure.

Proof. The largest possible $\Omega$ is the complement of $\bar{S}$ defined in Lemma 3. $\mathscr{D}(\Omega)$ is an invariant domain for the labelled observables and, if $\bar{S}$ has zero Lebesgue measure, it is dense in $\mathscr{H}$ by Lemma 1 . By Lemma 3, if $f \in D, f(x)=0(x \in \bar{S})$. Hence as in Lemma 1, if $\bar{S}$ has nonzero Lebesgue measure $D$ cannot be dense in $\mathscr{H}$.

\section{Topological semiinner product spaces}

Let $\Phi$ be a topological vector space on which a positive Hermitian form $(\phi, \psi)$ has been defined.

Definition $9 . \Phi$ is a topological semiinner product space if the mapping $\Phi \rightarrow \mathbf{C}$ defined by $\phi \rightarrow(\phi, \phi)$ is continuous. If further $(\phi, \phi)=0$ if and only if $\phi=0, \Phi$ is called a topological inner product space.

The natural domain for the kind of spectral theory we shall be presenting is the set of topological inner product spaces, but the theory may easily be adapted to semiinner product spaces. Since semiinner product spaces arise naturally in axiomatic field theory, it seems worthwhile presenting the theory so as to include this case. The Hilbert space formed by completion with respect to the inner product topology, after 
passage to the quotient space if necessary, will be denoted by $\mathscr{H}$. The canonical mapping $T: \Phi \rightarrow \mathscr{H}$ need now no longer be $1-1$. A topological semiinner product space may also be written as a triplet $\Phi \rightarrow \mathscr{H} \rightarrow \Phi^{\times}$, and $T^{\times}: \mathscr{H} \rightarrow \Phi^{\times}$will still be $1-1 . T^{\times} T$ will always be denoted by $\chi$.

Definition 10. Let $\Phi$ be a topological semiinner product space and let $N$ be the null space of $T$, then $\Phi / N$ with the quotient space topology has a natural structure as a topological inner product space, and will be called the associated topological inner product space to $\Phi$.

Note that $N=\{\phi \in \Phi \mid(\phi, \phi)=0\}=\{\phi \in \Phi \mid(\phi, \psi)=0(\psi \in \Phi)\}$. We shall show that the spectral theory of a semiinner product space can be reduced to that of the associated inner product space.

We shall always work with locally convex spaces, and we need to endow $\Phi^{\times}$with some notion of continuity. The exact notion does seem crucial provided that the continuity of $S: \Phi \rightarrow \Psi$ implies that of $S^{\times}: \Psi^{\times} \rightarrow \Phi^{\times}$for all locally convex space $\Phi$ and $\Psi$, and also that $\mathscr{H} \cong \mathscr{H}^{\times}$. For example, we could give each $\Phi^{\times}$the Mackey topology of the dual system or indeed its natural pseudotopology as a polynorm space [13]. We shall always suppose that the dual spaces have been given some suitable notion of continuity.

Definition 11. Let $A \in \mathscr{L}(\Phi)$ be the set of continuous linear operators on $\Phi$, then $A$ will be said to have a conjugate $A^{c}$ if there exists an $A^{c} \in \mathscr{L}(\Phi)$, such that $\left(A^{c} \phi, \psi\right)=(\phi, A \psi)(\phi, \psi \in \Phi)$. The set of operators with conjugates will be denoted by $\mathscr{L}^{c}(\Phi)$. If $A$ is a conjugate of itself, it is said to be real.

Note that conjugates are unique if and only if we are dealing with inner product spaces.

Proposition 16. Let $\Phi$ be a semiinner product space, $\Phi / N$ the associated inner product space, and $K$ the canonical projection $K: \Phi \rightarrow \Phi / N$ then if $A \in \mathscr{L}^{c}(\Phi)$, there exists an $\hat{A} \in \mathscr{L}^{c}(\Phi / N)$ such that

Proof.

$$
\hat{A} K=K A \text {. }
$$

$$
\left(A^{c} \phi, \psi\right)=(\phi, A \psi) \quad(\phi, \psi \in \Phi) .
$$

This implies that $A$ and $A^{c}$ leave $N$ invariant. Hence they define operators $\hat{A}$ and $\widehat{A^{c}}$ on $\Phi / N$, satisfying $\hat{A} K=K A$ and $\widehat{A^{c}} K=K A^{c}$. These must be continuous as $\Phi / N$ has the finest locally convex topology making $K$ continuous. Finally $\widehat{A^{c}}=\hat{A}^{c}$, so $\hat{A} \in \mathscr{L}^{c}(\Phi / N)$.

Proposition 17. If $A \in \mathscr{L}^{c}(\Phi)$, then $\chi A=A^{c \times} \chi$.

The proof is straightforward. If $\Phi$ is an inner product space, it may be identified with a subspace of $\Phi^{\times}$, so that $A^{c \times}$ is a continuous extension of $A$ to $\Phi^{\times}$. 
Definition 12. If $\gamma \in \mathscr{L}\left(\Phi, \Phi^{\times}\right)$, then we say $\gamma$ is self-adjoint if

$$
\langle\phi, \gamma \psi\rangle=\overline{\langle\psi, \gamma \phi}\rangle \quad(\phi, \psi \in \Phi) .
$$

We say that $\gamma$ is positive if $\langle\phi, \gamma \phi\rangle \geqq 0(\phi \in \Phi)$.

Proposition 18. $\gamma \in \mathscr{L}\left(\Phi, \Phi^{\times}\right)$is self-adjoint if and only if $\langle\phi, \gamma \phi\rangle$ is real $(\phi \in \Phi)$.

Proof. We have the identity:

$$
\begin{aligned}
4\langle\phi, \gamma \psi\rangle= & \langle\phi+\psi, \gamma \phi+\gamma \psi\rangle-\langle\phi-\psi, \gamma \phi-\gamma \psi\rangle \\
& -i\langle\phi+i \psi, \gamma \phi+i \gamma \psi\rangle+i\langle\phi-i \psi, \gamma \phi-i \gamma \psi\rangle .
\end{aligned}
$$

Note that $\chi$ is positive and hence self-adjoint.

If $A \in \mathscr{L}^{c}(\Phi)$ then $T A T^{-1}$ defines an operator on $\mathscr{H}$. This operator is densely defined and has an adjoint $\left(T A T^{-1}\right) * \supset T A^{c} T^{-1}$ which is also densely defined. Hence $T A T^{-1}$ has a closure, which we shall call the closure of $A$ in $\mathscr{H}$, and denote by $\bar{A}$. We shall consider a spectral theory for $A$, which is closely related to the spectral theory of $\bar{A}$ in $\mathscr{H}$. In view of the interpretation of the Dirac formalism in terms of rigged Hilbert spaces [1], we shall call the elements of $\Phi$ bra vectors and the elements of $\Phi^{\times}$ket vectors.

\section{Definition 13.}

a) An eigenbra of $A$ is an eigenvector of $A$.

b) An eigenket of $A$ is an eigenvector of $A^{c \times}$.

c) An eigenoperator $\gamma$ of $A$, corresponding to the operator eigenvalue $\lambda$, is a positive non-zero element of $\mathscr{L}\left(\Phi, \Phi^{\times}\right)$such that

$$
A^{c \times} \gamma=\gamma A=\lambda \gamma \text {. }
$$

If $\gamma$ is an eigenoperator of $A$ corresponding to the operator eigenvalue $\lambda$ and if $\gamma \phi \neq 0$, then $\gamma \phi$ is an eigenket of $A$ and $A^{c}$, corresponding to the eigenvalues $\lambda$ and $\bar{\lambda}$ respectively. If $f \in \Phi^{\times}$is an eigenket of $A$ and $A^{c}$ with eigenvalues $\lambda$ and $\bar{\lambda}$ respectively, then $\bar{f} \otimes f$ is an eigenoperator of $A$ with eigenvalue $\lambda$. A ket eigenvalue of a real operator is an operator eigenvalue if and only if it is real.

\section{Integral decompositions}

Definition 14. An integral decomposition [14] of $\Phi$ is a triplet $\{\gamma(z), Z, \mu\}$ such that:

1) $\gamma(z) \in \mathscr{L}\left(\Phi, \Phi^{\times}\right)$is a positive operator $(z \in Z)$.

2) $\mu$ is a positive regular Borel measure on a locally compact Hausdorff space $Z$.

3) $z \rightarrow\langle\phi, \gamma(z) \psi\rangle$ is $\mu$-integrable $(\phi, \psi \in Z)$.

4) $(\phi, \psi)=\int_{Z}\langle\phi, \gamma(z) \psi\rangle d \mu(z)(\phi, \psi \in Z)$. 
Definition 15. Let $\Phi$ be a topological semiinner product space; then a sandwich space for $\Phi$ is a pair $(S, \Psi)$ such that $\Psi$ is a topological semiinner product space, and $S$ a continuous inner product preserving mapping, $S: \Phi \rightarrow \Psi$ onto a dense subspace of $\Psi$.

We now have a canonical quintuplet of spaces

$$
\Phi \rightarrow \Psi \rightarrow \mathscr{H} \rightarrow \Psi^{\times} \rightarrow \Phi^{\times} .
$$

If $\Phi / N$ is the associated inner product space of $\Phi, K$ the canonical mapping $K: \Phi \rightarrow \Phi / N$ then $(K, \Phi / N)$ is a sandwich space for $\Phi$.

Proposition 19. Let $(S, \Psi)$ be a sandwich space for $\Phi$ and $\{\gamma(z), Z, \mu\}$ an integral decomposition of $\Psi$, then $\left\{S^{\times} \gamma(z) S, Z, \mu\right\}$ is an integral decomposition of $\Phi$.

The proof of this proposition is immediate and, together with Proposition 16, it allows the spectral theory of semiinner product spaces to be reduced to the corresponding theory for the associated inner product spaces.

Proposition 20. Let $\{\gamma(z), Z, \mu\}$ be an integral decomposition of $\Phi$, then there is a unique operator-valued measure $\mathbf{m}$ on $Z$ with values in $\mathscr{L}\left(\Phi, \Phi^{\times}\right)$and countably additive with respect to $\sigma\left(\mathscr{L}\left(\Phi, \Phi^{\times}\right), \Phi \otimes \Phi\right)$ satisfying

$$
\langle\phi, \mathbf{m}(\delta) \psi\rangle=\int_{\delta}\langle\phi, \gamma(z) \psi\rangle d \mu(z)
$$

for all Borel sets $\delta$ of $Z$ and all $\phi, \psi \in \Phi$.

Proof.

$$
0 \leqq \int_{\delta}\langle\phi, \gamma(z) \phi\rangle d \mu(z) \leqq(\phi, \phi) \quad(\phi \in \Phi)
$$

Let

$$
m(\delta, \phi, \psi)=\int_{\delta}\langle\phi, \gamma(z) \psi\rangle d \mu(z)
$$

then $|m(\delta, \phi, \psi)|^{2} \leqq m(\delta, \phi, \phi) m(\delta, \psi, \psi)$, the Cauchy-Schwarz inequality. Hence $|m(\delta, \phi, \psi)| \leqq\|\phi\|\|\psi\|$. Thus, for fixed $\psi$ and $\delta, m$ is a continuous antilinear functional on $\Phi$ and defines an element $m(\delta, \psi)$ of $\Phi^{\times}$. For fixed $\delta, m(\delta, \psi)$ is a continuous linear map $\Phi \rightarrow \Phi^{\times}$and defines an element $\mathbf{m}(\delta)$ of $\mathscr{L}\left(\Phi, \Phi^{\times}\right)$.

Now $\langle\phi, \mathbf{m}(\delta) \psi\rangle=\int_{\delta}\langle\phi, \gamma(z) \psi\rangle d \mu(z)$. The countable additivity of $\mathrm{m}$ with respect to $\sigma\left(\mathscr{L}\left(\Phi, \Phi^{\times}\right), \Phi \otimes \Phi\right)$ follows trivially.

We have in fact proved considerably more than was set out in the proposition, for we have shown the continuity of $\mathbf{m}(\delta)$ with respect to the topology defined on $\mathscr{L}\left(\Phi, \Phi^{\times}\right)$by the scalar product on $\Phi$. This may be best expressed by introducing the notion of a semispectral measure [15], [19]. A semispectral measure is an operator-valued measure $\delta \rightarrow B(\delta)$, whose values are bounded Hermitian operators on a Hilbert space, and satisfying $0 \leqq B(\delta) \leqq 1, B(\emptyset)=0$ and $B(Z)=1$. A theorem due to 
NeUMark [15] asserts that any semispectral measure $\delta \rightarrow B(\delta)$ may be extended to a spectral measure $\delta \rightarrow E(\delta)$ in an extension space $\mathfrak{g} \supset \mathscr{H}$. Let $P$ denote the orthogonal projection onto the subspace $\mathscr{H}$ of $\mathfrak{S}$; then $E(\delta)$ is an extension of $B(\delta)$ in the sense that $B(\delta) \subset P E(\delta)$. If we require that $\{E(\delta) x \mid x \in \mathscr{H}, \delta$ a Borel set of $Z\}$ is dense in $\mathfrak{G}$, then the extension is determined to within an isomorphism. We now have

Proposition 21. Given an integral decomposition of a locally convex semiinner product space $\Phi,\{\gamma(z), Z, \mu\}$, there is a unique semispectral measure, $\delta \rightarrow B(\delta)$, defined on $\mathscr{H}$ and satisfying $(T \phi, B(\delta) T \psi)$ $=\langle\phi, \mathbf{m}(\delta) \psi\rangle=\int_{\delta}\langle\phi, \gamma(z) \psi\rangle d \mu(z) \quad(\phi, \psi \in \Phi)$.

Proof. The uniqueness of $B(\delta)$ follows because it is defined on the dense subspace $T(\Phi)$ of $\mathscr{H}$ by the relation

$$
(T \phi, B(\delta) \psi)=\langle\phi, \mathbf{m}(\delta) \psi\rangle \quad(\phi, \psi \in \Phi) .
$$

Clearly $0 \leqq B(\delta) \leqq 1, B(\emptyset)=0$ and $B(Z)=1$, so we do have a semispectral measure.

Definition 16. If $A \in \mathscr{L}^{c}(\Phi)$, then $\Phi$ has an integral A-eigendecomposition, if it has an integral decomposition with $\gamma(z)$ either 0 or an eigenoperator of $A(z \in Z)$. It has a real integral $A$-eigendecomposition, if it has an integral decomposition $\{\gamma(\lambda), \mathbf{R}, \mu\}$, such that

$$
A^{c \times} \gamma(\lambda)=\gamma(\lambda) A=\lambda \gamma(\lambda) \quad(\lambda \in \mathbf{R}) .
$$

Proposition 22. If $A \in \mathscr{L}^{c}(\Phi)$ and $\Phi$ has an integral $A$-eigendecomposition, then $\bar{A}$, the closure of $A$ in $\mathscr{H}$, is subnormal and formally normal.

Proof. Suppose that $A^{c \times} \gamma(z)=\gamma(z) A=f(z) \gamma(z)$, and let $B$ be the semispectral measure defined above. Consider the operator $C$ on $\mathscr{H}$ defined by:

$$
\begin{aligned}
D(C) & =\left\{\left.x \in \mathscr{H}\left|\int\right| f\right|^{2} d(x, B x)<\infty\right\} \\
C x & =\int f d B x \quad(x \in D(C)) .
\end{aligned}
$$

$C$ is closed since $\delta \rightarrow B(\delta)$ is a semispectral measure, and there is an extension of $B$ to a spectral measure in an extension space $\mathfrak{H} \supset \mathscr{H}$ [15]. The corresponding extension of $C$ is normal, so that $C$ is subnormal. Now

$$
(A \phi, A \phi)=\int|f(z)|^{2}\langle\phi, \gamma(z) \phi\rangle d \mu(z)<\infty,
$$

so that $T \phi \in D(C)(\phi \in \Phi)$.

$$
(T \phi, C T \psi)=\int f d(T \phi, B T \psi)=\int f(z)\langle\phi, \gamma(z) \psi\rangle d \mu(z) .
$$

Hence $(T \phi, C T \psi)=(\phi, A \psi)(\phi, \psi \in \Phi)$, which implies that $C$ is an extension of $A$ to $\mathscr{H}$. But $C$ is closed, hence $C \supset \bar{A}$, and $\bar{A}$ is subnormal as required. It remains to show that $\bar{A}$ is formally normal, we recall that a closed operator $\bar{A}$ on $\mathscr{H}$ is formally normal if $D(\bar{A}) \subset D\left(\bar{A}^{*}\right)$ and if $\|\bar{A} x\|=\left\|\bar{A}^{*} x\right\|(x \in D(\bar{A}))$. 
Now

Similarly

$$
\begin{aligned}
(A \phi, A \psi) & =\int\langle A \phi, \gamma(z) A \psi\rangle d \mu(z), \\
(A \phi, A \psi) & =\int f(z)\langle A \phi, \gamma(z) \psi\rangle d \mu(z) \\
& =\int|f(z)|^{2}\langle\phi, \gamma(z) \psi\rangle d \mu(z) .
\end{aligned}
$$

$$
\left(A^{c} \phi, A^{c} \psi\right)=\int|f(z)|^{2}\langle\phi, \gamma(z) \psi\rangle d \mu(z) .
$$

Hence $(A \phi, A \psi)=\left(A^{c} \phi, A^{c} \psi\right)$, and so $\|\bar{A} T \phi\|=\left\|\bar{A}^{*} T \phi\right\|,(\phi \in \Phi)$. Then by taking closures $\|\bar{A} x\|=\left\|\bar{A}^{*} x\right\|(x \in D(\bar{A}))$, and $D(\bar{A}) \subset D\left(\bar{A}^{*}\right)$, so that $\bar{A}$ is indeed formally normal.

Proposition 23. If $A \in \mathscr{L}^{c}(\Phi)$ has a real integral eigendecomposition in $\Phi$, then $A$ is real.

Proof. We proceed as before, noting that $\bar{A}$ now has a self-adjoint extension, so that $A$ is real.

\section{Spectral theory}

This last section is devoted to showing that, in a rigged Hilbert space, the converse of the last two propositions is also valid. In fact the results hold a little more generally, being valid if the canonical mapping $T: \Phi \rightarrow \mathscr{H}$ is nuclear. The basic lemma of the theory, which is due to GARDING [16], is the following:

Lemma 4. Let $\Phi$ be a locally convex space, and let $T$ be a nuclear mapping into a direct integral of Hilbert spaces,

$$
T: \Phi \rightarrow \mathscr{H}=\int^{\oplus} \mathscr{H}(z) d \mu(z)
$$

then there exist nuclear mappings $T(z): \Phi \rightarrow \mathscr{H}(z)(z \in Z)$, satisfying $T \phi=\int T(z) \phi d \mu(z) .(\phi \in \Phi)$.

The following pair of lemmas is designed simply to cope with the case in which $\Phi$ is not separable. For convenience, we shall give the theory in a form, which applies only to inner product spaces.

Lemma 5. Let $E$ be a locally convex space, $T$ a nuclear mapping of $E$ into a Hilbert space $\mathscr{H}$. Then there exists a separable Banach space $F$ and mappings $T_{1}: E \rightarrow F, T_{2}: F \rightarrow \mathscr{H}$ such that $T_{1}$ is continuous, $T_{2}$ is nuclear and $T=T_{2} T_{1}$.

Proof. As $T$ is nuclear, it has the form (see definition 6)

$$
T x=\sum_{k=1}^{\infty} \lambda_{k}\left\langle x, x_{k}^{\prime}\right\rangle y_{k} \quad(x \in E)
$$

where $\left(x_{k}^{\prime}\right)$ is bounded in $E_{A}^{\prime},\left(y_{k}\right)$ is bounded in $\mathscr{H}$, and where $\sum_{k=1}^{\infty}\left|\lambda_{k}\right|<\infty$. We may in fact suppose further that $x_{k}^{\prime} \rightarrow 0$ in $E_{A}^{\prime}$ [6]. Let $c_{0}$ denote the usual Banach space of sequences of complex numbers tending to zero, then $\left(\left\langle x, x_{k}^{\prime}\right\rangle\right) \in c_{0}(x \in E)$. Define a linear mapping $T_{1}: E \rightarrow c_{0}$ by setting 
$T_{1} x=\left(\left\langle x, x_{k}^{\prime}\right\rangle\right) . T_{1}$ is continuous since $A$ is equicontinuous. Define a mapping $T_{2}: c_{0} \rightarrow \mathscr{H}$ by setting $T_{2}\left(\alpha_{k}\right)=\sum_{k=1}^{\infty} \lambda_{k} \alpha_{k} y_{k}\left(\left(\alpha_{k}\right) \in c_{0}\right) . T_{2}$ is nuclear and $T_{2} T_{1}=T . c_{0}$ is separable so we may take $F=c_{0}$.

Note that if $T$ is $1-1, T_{1}$ must also be $1-1$.

Lemma 6. Let $E$ have a locally convex topology $\tau$ and suppose that $T: E \rightarrow \mathscr{H}$ is $1-1$ and nuclear and that $A_{i}: E \rightarrow E$ are continuous linear mappings $(i=1,2, \ldots, n)$. Then there exist topologies $\tau_{1}$ and $\tau_{2}$ such that

a) $E$ is a separable metric space under $\tau_{1}$ or $\tau_{2}$.

b) $\tau \leqq \tau_{1} \leqq \tau_{2} \leqq \tau_{\mathscr{H}}\left(\tau_{\mathscr{H}}\right.$ the Hilbert space topology on $\left.E\right)$.

c) $E_{\tau_{2}} \rightarrow \mathscr{H}$ is nuclear.

d) $A_{i}: E_{\tau_{1}} \rightarrow E_{\tau_{2}}$ is continuous $(i=1,2, \ldots, n)$.

Proof. Take $F$ as in Lemma 5, then $T_{1}$ identifies $E$ with a subspace of $F$. Let $\tau_{2}$ be the induced topology. Put $A_{0}=I: E \rightarrow E$, and let $\tau_{1}$ be the initial topology on $E$ with respect to the mappings $A_{i}: E \rightarrow E_{\tau_{2}}$ $(i=0,1,2, \ldots, n)$. By Proposition $6, E_{\tau_{1}}$ is isomorphic to a subspace of $\prod_{i=0}^{n} E_{\tau_{2}}$ and is thus a separable metric space. The other conditions may be verified without difficulty.

Theorem 3. Let $\Phi$ be a locally convex inner product space with a nuclear canonical mapping $T$, and let $A \in \mathscr{L}^{c}(\Phi)$ have a closure $\bar{A}$ which is subnormal and formally normal. Then $\Phi$ has an integral $A$-eigendecomposition.

Proof. There exists a Hilbert space $\mathscr{H}_{1}$, a projection $P$ and a normal operator $A_{1}$, such that $\mathscr{H}=P \mathscr{H}_{1}$ and $\bar{A}=P A_{1}$. Take a direct integral decomposition of $\mathscr{H}_{1}$ diagonalizing $A_{1}$.

$$
\begin{aligned}
\mathscr{H}_{1} & =\int^{\oplus} \mathscr{H}_{1}(z) d \mu(z) \\
\left(A_{1} x\right)(z) & =0 z x(z) \quad\left(x \in D\left(A_{1}\right)\right)^{1} .
\end{aligned}
$$

Apply Lemma 4 with $\Phi$ considered as embedded in $\mathscr{H}_{1}$ and $T: \Phi \rightarrow \mathscr{H}_{1}$. Define $\gamma(z): \Phi \rightarrow \Phi^{\times}$by $\gamma(z)=T(z)^{\times} T(z)$, where $T(z)$ is defined as in Lemma $4, T(z): E \rightarrow \mathscr{H}_{1}(z)$. Then

and

$$
\langle\phi, \gamma(z) \psi\rangle=\left\langle\phi, T(z)^{\times} T(z) \psi\right\rangle=(T(z) \phi, T(z) \psi)_{z},
$$

$$
\langle\phi, \gamma(z) \phi\rangle=(T(z) \phi, T(z) \phi)_{z} \geqq 0 \quad(\phi \in \Phi)
$$

so that $\gamma(z)$ is positive. Further

$$
(\phi, \psi)=\int([T \phi](z),[T \psi](z))_{z} d \mu(z)=\int(T(z) \phi, T(z) \psi)_{z} d \mu(z) .
$$

Hence

$$
(\phi, \psi)=\int\langle\phi, \gamma(z) \psi\rangle d \mu(z) \quad(\phi, \psi \in \Phi) .
$$

1 The symbol $={ }^{0}$ is used to denote equality $\mu$-almost everywhere. 
Now

thus

$$
T A \phi=\bar{A} T \phi=A_{1} T \phi
$$

$$
T(z)[A \phi]={ }^{0}\left[A_{1} T \phi\right](z)={ }^{0} z[T \phi](z)={ }^{0} z T(z) \phi .
$$

The exceptional set depends, in the first instance, on the $\phi$ chosen, but applying Lemma 6 to the case of a single operator $A$, let $\left\{\phi_{i}\right\}, i=1,2, \ldots$, be a countable dense set in the $\tau_{1}$ topology on $\Phi$. Now $T(z)\left[A \phi_{i}\right]$ $=z T(z) \phi_{i}(i=1,2, \ldots)$ unless $z$ lies in some $\mu$-null set $N$. Further, by c) of Lemma 6, we may suppose that $T(z)$ is continuous when $\Phi$ has the $\tau_{2}$ topology. Given $\phi \in \Phi$, there exists $\left(\phi_{i_{n}}\right)$ such that $\phi_{i_{n}} \rightarrow \phi$ in the $\tau_{1}$ topology as $n \rightarrow \infty$. Proceeding to the limit, we have

$$
T(z)[A \phi]=z T(z) \phi \quad(\phi \in \Phi, z \notin N) .
$$

Now since $A_{1}$ is normal $D\left(A_{1}^{*}\right) \supset D\left(A_{1}\right) \supset \Phi$. Hence

$$
\left(T \phi, T A^{c} \psi\right)=(T A \phi, T \psi)=\left(A_{1} T \phi, T \psi\right)=\left(T \phi, A_{1}^{*} T \psi\right)
$$

so that $A_{1}^{*} T \psi-T A^{c} \psi \in \Phi \perp$. But, since $\bar{A}$ is formally normal, $\left\|A_{1}^{*} T \psi\right\|$ $=\left\|A_{1} T \psi\right\|=\|T A \psi\|=\left\|T A^{c} \psi\right\|$. Hence $T A^{c} \phi=A_{1}^{*} T \phi$, so that $T(z) A^{c} \phi={ }^{0}\left[A_{1}^{*} T \phi\right](z)={ }^{0} \bar{z} T(z) \phi(\phi \in \Phi)$, where, as above, we may show that the null set may be chosen to be independent of $\phi$. We now put $T(z)=0$ in the exceptional sets, so that we may suppose that $T(z) A$ $=z T(z)$ and $T(z) A^{c}=\bar{z} T(z)(z \in Z)$. It follows at once that $A^{c \times} \gamma(z)$ $=z \gamma(z)=\gamma(z) A$, completing the proof of the theorem.

Corollary. Under the conditions of the theorem, an operator $A \in \mathscr{L}(\Phi)$ has a real integral $A$-eigendecomposition if and only if $A$ is real.

Proof. If $A$ is real, $\bar{A}$ is symmetric and hence has a self-adjoint extension. The converse is contained in Proposition 23.

The next result relates to the uniqueness of integral eigendecompositions. We cannot expect true uniqueness even for eigendecompositions $\{\gamma(z), \mathbf{C}, \mu\}$ where $A^{c \times} \gamma(z)=\gamma(z) A=z \gamma(z)(z \in \mathbf{C})$. However, we shall say that two such eigendecompositions $\left\{\gamma_{1}(z), \mathbf{C}, \mu_{1}\right\}$ and $\left\{\gamma_{2}(z), \mathbf{C}, \mu_{2}\right\}$ are equivalent [14], if there exists a regular positive Borel measure $\mu$ and measurable functions $f_{1}$ and $f_{2}$ such that $\mu_{1}=f_{1} \mu$, $\mu_{2}=f_{2} \mu$ and $f_{1} \gamma_{1}(z)=f_{2} \gamma_{2}(z)$ except on a set of $\mu$-measure zero.

Proposition 24. Let $\Phi$ be a locally convex inner product space with a nuclear embedding; then a real operator $A \in \mathscr{L}(\Phi)$ has a real $A$-eigendecomposition, unique to within equivalence, if and only if $\bar{A}$ is maximal Hermitian.

Proof. By Proposition 21, integral decompositions determine unique semispectral measures. Clearly two integral eigendecompositions are equivalent if and only if they determine the same semispectral measure over C. Hence $\Phi$ has a real $A$-eigendecomposition, unique to within equivalence, if and only if $\bar{A}$ has a unique attached generalized spectral resolution, i.e. if and only if $\bar{A}$ is maximal Hermitian. 
For the case of a continuous observable [1] in quantum mechanics, where we know we have a self-adjoint extension, this condition reduces to the observable's being essentially self-adjoint on $\Phi$. This raises the question of whether the usual observables are essentially self-adjoint on bra space. Here we content ourselves with the remark that the Hamiltonian is essentially self-adjoint on bra space if and only if the potential is everywhere infinitely differentiable [8]. The change from an integral eigendecomposition to an equivalent one corresponds to a change in weight function of the representation.

As the Gårding lemma, Lemma 4, depends only on the existence of a direct integral decomposition of Hilbert spaces, it is clear how to proceed to try and extend Theorem 3 to the case where we have a set of operators with commuting spectra. A weakened form of this theorem is always valid.

Definition 17. If $\mathscr{N} \subset \mathscr{L}^{c}(\Phi)$, then an integral decomposition $\{\gamma(z), Z, \mu\}$ is an $\mathscr{N}$-almost eigendecomposition if

$$
A^{c \times} \gamma(z) \phi={ }^{0} \gamma(z) A \phi={ }^{0} f(z) \gamma(z) \phi \quad(A \in \mathscr{N}, \phi \in \Phi) .
$$

In this definition the null set is allowed to depend on $\phi$ and $A$. For the remainder of this paper we shall suppose that $\Phi$ is a locally convex inner product space with a nuclear embedding.

Theorem 4. Let $\mathscr{N} \subset \mathscr{L}^{c}(\Phi)$, then $\Phi$ has an $\mathscr{N}$-almost eigendecomposition, if and only if $\mathscr{N}$ is formally normal, and has an extension $\mathscr{M}$ consisting of normal operators with commuting spectral resolutions in some extension space. In particular if $\mathscr{N}$ is set of self-adjoint operators, then $\Phi$ has an $\mathscr{N}$-almost eigendecomposition if and only if $\mathscr{N}$ is Abelian.

Proof. The procedure followed in Proposition 22 applies equally well to any $\mathscr{N}$-almost eigendecomposition. For the converse, we take a direct integral decomposition of the extension space, which decomposes $\mathscr{M}$ [17], and apply Lemma 4. We may now proceed as in Theorem 3, but do not need to attempt to eliminate the dependence of the null set on $A$ and $\phi$.

If we want true $\mathscr{N}$-eigendecompositions, then we seem to need some countability conditions on $\mathscr{N}$ and $\Phi$.

Lemma 7. Let $\Phi$ be a rigged Hilbert space constructed as in Proposition 11, and let $A_{i} \in \mathscr{L}^{c}(\Phi)(i=1,2, \ldots)$, then $\Phi$ has a coarser topology as a separable rigged Hilbert space such that $A_{i} \in \mathscr{L}^{c}(\Phi)$ $(i=1,2, \ldots)$.

Proof. By Theorem 1, there exists a real $A \in \mathscr{L}(\Phi)$, which guarantees the nuclearity of $\Phi$. Now take the semigroup with unit generated by $\left\{A, A_{i}, A_{i}^{c}, i=1,2, \ldots\right\}$ and give $\Phi$ the initial topology with respect to this semigroup of mappings $\Phi \rightarrow \mathscr{H}$. The resulting topology is nuclear by Theorem 1 and is metrizable. Hence $\Phi$ is separable. (Its completion 
is a Fréchet Montel space and is thus separable [3].) Further $A_{i} \in \mathscr{L}^{c}(\Phi)$ in this new topology by Proposition 9.

Theorem 5. Let $\mathscr{N} \subset \mathscr{L}^{c}(\Phi)$ and suppose either

a) $\mathscr{N}$ is a finite set of operators.

b) $\mathscr{N}$ is a countable set and $\Phi$ is separable.

c) $\mathscr{N}$ is a countable set and $\Phi$ is a nuclear space constructed as in Proposition 11.

Then the conclusions of Theorem 4 hold without the restriction to almost eigendecompositions.

Proof. The point at issue is the dependence of the null set in Theorem 4 on $\phi$ and $A . \mathscr{N}$ is at most a countable set, so the dependence on $A$ may be eliminated. In case a) we can eliminate the dependence on $\phi$ by using Lemma 6 and arguing as in Theorem 3 . In case b) we can proceed directly without using Lemma 6 . Case c) may be reduced to case b) by using Lemma 7.

Proposition 25. If $\mathscr{N}$ is a set of self-adjoint operators, then $\overline{\mathscr{N}}^{\prime \prime}$ the $W^{*}$-algebra generated by $\mathscr{N}$, is maximal Abelian [18] if and only if $\Phi$ has an $\mathscr{N}$-almost eigendecomposition $\{\gamma(z), Z, \mu\}$ where the non-zero $\gamma(z)$ have rank 1 .

Proof. If $\overline{\mathscr{N}}^{\prime \prime}$ is maximal abelian, then we can decompose $\mathscr{H}$ as a direct integral $\mathscr{H}=\int^{\oplus} \mathscr{H}(z) d \mu(z)$ in such a way that $\overline{\mathscr{N}}^{\prime \prime}$ is the set of bounded diagonalizable operators and $\operatorname{dim} \mathscr{H}(z)={ }^{0} 1$. Defining the $T(z)$ as in Lemma 4, and putting $T(z)=0$ if $\operatorname{dim} \mathscr{H}(z) \neq 1$, we have $\gamma(z)=T(z)^{\times} T(z)$ with rank 1 if it is non-zero, in which case it is also an $\mathscr{N}$-almost eigenoperator. Conversely, given $\gamma(z)$, let $\mathscr{H}(z)=\gamma(z) \Phi$ with an inner product $(\phi(z), \psi(z))_{z}=\langle\phi, \gamma(z) \psi\rangle$, then $\mathscr{H}=\int^{\oplus} \mathscr{H}(z) d \mu(z)$ and $\mathscr{N}^{\prime \prime}$ corresponds to the bounded diagonalizable operators. Hence $\widetilde{N}^{\prime \prime}$ is indeed maximal Abelian.

This result applied to the case where $\overline{\mathscr{N}}$ is a finite complete set of commuting observables is sufficient to give the Dirac type of representation as a function space over the range of the simultaneous eigenvalues [1].

There are many different possible approaches to proving the existence of some form of generalized eigenvector expansions which have been given in the literature. We conclude by comparing the results we have obtained with those obtained by others in the hope that this may be of some assistance to the reader in finding his way through the literature. However we make no pretence of completeness in citing the sources.

The approach to spectral theory using topological semiinner product spaces is the author's own, but we have followed Foras [14] in using integral decompositions and eigenoperators. However we have followed MAURIN [16] in using Lemma 4 and direct integrals of Hilbert spaces, 
rather than use the integral representations of vector-valued measures of bounded variation favoured by Foras [19]. In the work of MAUTNER [20] with his eigenfunction expansions and GARDING [22], [23] with his Carleman kernels, we only have generalized eigenvectors in a sense akin to our almost eigendecompositions, Definition 17. GEL'FAND and KostyuCEnko [24] were the first to demonstrate the existence of expansions using generalized eigenvectors in the sense of our eigenkets, but they use the notion of differentiation of vector-valued functions. We, like ForAs, have preferred to avoid this notion, so as to allow integral eigendecompositions over general locally compact spaces $Z$. Again following FoIAs, we have presented the theory in a way which takes full advantage of the Neumark extension of semispectral measures to spectral measures [15]. Using Lemmas 5 and 6, we have however succeeded in avoiding the separability hypothesis on $\Phi$ made by Foras [14] and by GEL'FAND and VILENKIN [11], although we are still forced to introduce some countability hypotheses in Theorem 6 .

Acknowledgements. The author wishes to acknowledge the help and encouragement given him by his supervisors, Dr. R. J. Eden and Professor R. Jost, during the course of this work. He is particularly grateful to Professor Jost for allowing him to enjoy the hospitality of the Institut für Theoretische Physik, ETH, Zürich, for three semesters. The author is also duly grateful for the financial support of a D.S.I.R. grant and latterly of a Research Fellowship at Emmanuel College, Cambridge.

\section{References}

[1] Roberts, J. E.: J. Math. Phys. 7, $1097-1104$ (1966).

[2] Bourbaki, N.: Topologie générale, 3rd ed. Paris: Hermann 1961.

[3] Köтне, G.: Topologische lineare Räume. Berlin, Göttingen, Heidelberg: Springer 1960 .

[4] Grothendieck, A.: Espaces vectoriels topologiques, 3rd ed. Sociedade de Matemática de Sao Paulo, 1964.

[5] BourbaKi, N.: Espaces vectoriels topologiques. Paris: Hermann 1964.

[6] Grothendieck, A.: Produits tensoriels topologiques et espaces nucléaires. Mem. Am. Math. Soc. No. 16, AMS, Providence, R.I., 1955.

[7] Schwartz, L.: Produits tensoriels topologiques d'espaces vectoriels topologiques. Espaces vectoriels topologiques nucléaires. Applications. Séminaire, Institut Henri Poincaré, Paris 1954.

[8] Roberts, J. E.: Cambridge Thesis, 1965 (unpublished).

[9] Murray, F. J., and J. von Neumann: Ann. Math. 37, 116-229 (1936).

[10] Gel'fand, I. M., and G. E. SIllov: Generalized functions 2. Moscow : Fizmatgiz 1958.

[11] 一, and N. YA. VILENkin: Generalized functions 4. Moscow: Fizmatgiz 1961.

[12] Schwartz, L.: Théorie des distributions, 2nd ed. Paris: Hermann 1957/59.

[13] Marinescu, G.: Espaces vectoriels pseudotopologiques et théorie des distributions. Berlin: VEB Deutscher Verlag der Wissenschaften 1963.

[14] Foras, C.: Revue de math. pures et appl. 7, 241-282, and 571-602 (1962).

[15] Sz.-NAGY, B.: Extensions of linear transformations on Hilbert space which extend beyond this space. (Appendix to F. RIEsz and B. Sz.-NAGY 'Functional analysis'). New York: Ungar 1960. 
[16] Maurin, K.: Bull. Acad. Pol. Sci. Sér. Sci. Math. 7, 470-479 (1959).

[17] Nussbaum, A. E.: Duke Math. J. 31, 33-44 (1964).

[18] Drxmier, J.: Les algèbres d'opérateurs dans l'espace Hilbertien. Paris: Gauthier-Villars 1957.

[19] Foras, C.: Acta Sci. Math. (Szeged) 20, 117-155 (1959).

[20] Mautner: F. I.: Proc. Nat. Acad. Sci. U.S. 39, 49-53 (1953).

[21] Bade, W. G., and J. T. Schwartz: Proc. Nat. Acad. Sci. U.S. 42, 519-525 (1956).

[22] GÅrding: L.: Kungl. Fysiografiska Sällskapets i Lund, Förhandlingar 24, 190-206 (1954).

[23] -Lectures notes, University of Maryland, 1954.

[24] Gel'fand, I. M., and A. G. Kostyučenko: Dokl. Akad. Nauk SSSR (N.S.) $103,345-352$ (1955).

[25] —, and G. E. Šrov: J. de Math. (Liouville) Sér. 9, 35, 383-413 (1956). 